\title{
Lithium Abundances in Fast Rotating Bright Giant Stars
}

\author{
J.D.Jr do Nascimento ${ }^{1}$, A. Lèbre ${ }^{2}$, R. Konstantinova-Antova ${ }^{3}$ and J. R. \\ De Medeiros ${ }^{1}$ \\ 1-Departamento de Física Teorica e Experimental, Universidade Federal \\ do Rio Grande do Norte, 59072-970 Natal, R.N., Brazil; ${ }^{2}-$ GRAAL, \\ UMR 5024 ISTEEM/CNRS, CC 072, Université Montpellier II, \\ F-34095 Montpellier Cedex, France; ${ }^{3}$ Institute of Astronomy, 72 \\ Tsarigradsko shose, BG-1784 Sofia, Bulgaria
}

\begin{abstract}
We present the results of high resolution spectroscopic observations of $\mathrm{Li}$ I resonance doublet at $\lambda 6707.8 \AA$ for fast rotating single stars of luminosity class II and Ib. We present a discussion on the link between rotation and $\mathrm{Li}$ content in intermediate mass giant stars, with emphasis on their evolutionary status. At least one of the observed stars, HD 232862, a G8II with an unusual vsini of $20 \mathrm{~km} / \mathrm{s}$, present a Li-rich behavior.
\end{abstract}

\section{The Problem and Observations}

This work brings the first results of a large observational campaign to determine $\mathrm{Li}$, rotation and $\mathrm{CNO}$ abundances for a sample of single bright giants and Ib supergiants, along the spectral region $\mathrm{F}, \mathrm{G}$ and $\mathrm{K}$. Measurements of $\mathrm{Li}$ and CNO surface content of evolved stars is of paramount importance to test quantitative and qualitative theoretical predictions of the effects of nucleosynthesis and subsequent mixing events on the stellar surface abundances.

A survey of bright giants and Ib supergiants along the spectral region $F, G$ and $\mathrm{K}$, has been carried out to study the possible link between the rotational behavior and CNO abundances (do Nascimento \& De Medeiros 2003). The sample of about 300 bona fide stars, is complete to apparent visual magnitude of about 8.0. The evolutionary status was determined from HIPPARCOS (ESA 1997). All the stars have $v \sin i$ velocity available in the Catalog of rotational velocities by De Medeiros and Mayor (1999) and by De Medeiros et al. (2002). Spectroscopic data have been acquired using the spectrographs FEROS at ESO/Chile, AURELIE at OHP/France and the coudé spectrograph at LNA/Brazil.

\section{First Results: A Quick-Look}

During inspection of the data we spotted an extremely strong $\lambda 6707.8 \AA$ feature in HD 232862. In order to perform a spectrum synthesis, a grid of stellar atmospheric models from Kurucz et al. (1993), has been cubically interpolated and then used as input to the revised version of the code MOOG (Sneden 1973) with lines taken from the VALD database (Piskunov et al. 1995). We obtained the best $(\mathrm{O}-\mathrm{C})$ residuals for $\mathrm{T}_{\text {eff }}=5200 \mathrm{~K}$ (Figure 1$)$. The obtained abundance 


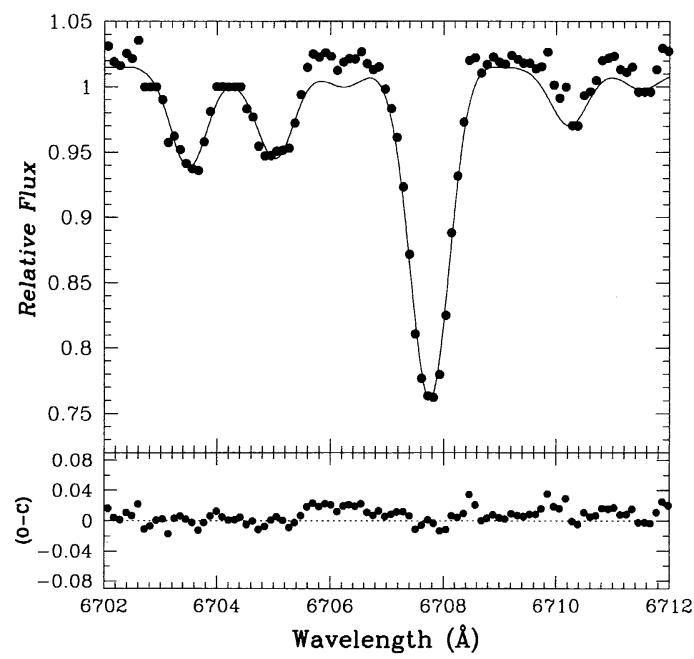

Figure 1. Comparison of the synthetic and observed spectra for HD 232862 around $\lambda 6707.8 \AA$ region. Continuous line and points represent the synthetic and observed spectra, respectively.

for this best value of $\mathrm{T}_{\text {eff }}$ is $\log (L i)=2.9$ The high $\mathrm{Li}$ abundance obtained for HD 232862, indicates that this G8II bright giant has a Li content far in excess of the expected value for its spectral type. Nevertheless a interesting point here concerns the mass for this star. Until now single late-G and $\mathrm{K} \mathrm{Li}$-rich giants reported in the literature are almost low mass stars (Drake et al. 2002). In contrast, HD 232862 seems to be a moderate mass star. Masses of bright giant stars range from about 3 to 9 solar mass. The present results indicate that the root-causes of the Li-rich property in single late- $\mathrm{G}$ and $\mathrm{K}$ evolved stars also operate in stars of different luminosity classes and mass intervals.

\section{References}

Drake, N. A., de la Reza, R., Silva, L., \& Lambert, D. L. 2002, AJ 123, 2703

De Medeiros, J. R., Mayor, M. 1999, A\&AS 139, 433

De Medeiros, J. R., Udry, S., Burki, G., Mayor, M. 2002, A\&A 395, 97

do Nascimento, J. D., Jr., De Medeiros, J. R. 2003, proceedings CNO in the Universe,

C. Charbonnel, D. Schaerer and G. Meynet eds., ASP Conf. Ser. 119

ESA 1997, The Hipparcos and Tycho Catalogues, ESA SP-1200

Kurucz, R. L. 1993, CD-ROMs, ATLAS9 Stellar Atmospheres Programs and $2 \mathrm{~km} \mathrm{~s}^{-1}$

Grid (Cambridge: Smithsonian Astrophys. Obs.)

Piskunov, N. E., Kupka, F., Ryabchikova, T. A., Weiss, W. W., \& Jeffery, C. S. 1995, A\&AS 112, 525

Sneden, C. 1973, Ph.D. Thesis, University of Texas 